

www.revistahorizontes.org
Horizontes. Revista de Investigación en Ciencias de la Educación Https://doi.org/10.33996/revistahorizontes.v4i14.98

Abril - Junio 2020

Volumen 4 / No. 14

ISSN: 2616 - 7964

ISSN-L: $2616-7964$ pp. $139-148$

\title{
Uso de la tecnología en las aulas universitarias, ¿una utopía en la era de la información?
}

\author{
Use of technology in university classrooms, is a utopia in the information age? \\ Uso da tecnologia nas salas de aula das universidades, uma utopia na era da \\ informação?
}

\section{Sylvia Ibeth Tapia Tapia ${ }^{1}$}

sitapia@uce.edu.ec

Código ORCID: 0000-0003-1760-3628

\author{
Andrea Cecibel Campoverde Castillo ${ }^{2}$ \\ andrea.c.campoverde@unl.edu.ec \\ Código ORCID: 0000-0003-4847-4507
}

\author{
Karla Stefanie Medina Aguilar² \\ karla.s.medina@unl.edu.ec \\ Código ORCID: 0000-0003-3871-7608 \\ Universidad Central del Ecuador, Ecuador ${ }^{1}$ \\ Universidad Nacional de Loja, Ecuador ${ }^{2}$
}

Recibido diciembre 2019 | Revisado enero 2020 | Publicado 01 de abril 2020

\section{RESUMEN}

En esta era tecnológica todo tipo de información está al alcance de un dispositivo electrónico y el Internet se ha vuelto indispensable en la educación; ya no se discute la efectividad de las TIC en los procesos de formación académica. La universidad está sujeta a la constante modernización de sus paradigmas pedagógicos y en esta ola de avance educativo el uso de la tecnología para fortalecer y consolidar los conocimientos en los docentes va teniendo cada día más impulso; no obstante, su uso no se vuelve una práctica común entre ellos. La finalidad de este estudio fue analizar mediante una reflexión sobre el uso de la tecnología como parte de la pedagogía universitaria. Consecuentemente, se realizó una revisión bibliográfica sobre la temática. Se pudo concluir que todavía falta aprender sobre la relación entre tecnología y educación superior, usar las TIC por si solas no garantiza el éxito, es imperioso vincularlas a estructuras pedagógicas bien definidas, un docente comprometido es la clave.

Palabras clave: Tecnología; universidad; docentes; estudiantes; procesos pedagógicos

\begin{abstract}
In this technological age, all kinds of information are within the reach of an electronic device and the Internet has become indispensable in education; the effectiveness of ICT in academic training processes is no longer discussed. The university is subject to the constant modernization of its pedagogical paradigms and in this wave of educational advance the use of technology to strengthen and consolidate the knowledge of teachers is getting more and more momentum every day; however, its use does not become a common practice among them. The purpose of this study was to analyze through a reflection on the use of technology as part of university pedagogy. Consequently, a bibliographic review on the subject was carried out. It was concluded that learning about the relationship between technology and higher education still remains, using ICT alone does not guarantee success, it is imperative to link them to well-defined pedagogical structures, a committed teacher is the key.
\end{abstract}

Key Words: Technology; university; teachers; students; pedagogical processes 


\section{RESUMO}

Nesta era tecnológica, todo tipo de informação está ao alcance de um dispositivo eletrônico e a Internet se tornou indispensável na educação; A eficácia das TIC nos processos de formação acadêmica não é mais discutida. A universidade está sujeita à constante modernização de seus paradigmas pedagógicos e, nessa onda de avanço educacional, o uso da tecnologia para fortalecer e consolidar o conhecimento dos professores ganha cada vez mais força todos os dias; no entanto, seu uso não se torna uma prática comum entre eles. 0 objetivo deste estudo foi analisar através de uma reflexão sobre o uso da tecnologia como parte da pedagogia universitária. Consequentemente, foi realizada uma revisão bibliográfica sobre o assunto. Concluiu-se que o aprendizado sobre a relação entre tecnologia e ensino superior ainda permanece; somente o uso das TIC não garante o sucesso; é imperativo vinculá-las a estruturas pedagógicas bem definidas; um professor comprometido é a chave.

Palavras-chave: Tecnologia; Faculdade; professores; estudantes; processos pedagógicos

\section{INTRODUCCIÓN}

Actualmente la tecnología se ha vuelto parte de la cotidianidad de los jóvenes universitarios quienes están interesados en los avances continuos de ésta, y tomando en cuenta que serán los futuros profesionales al servicio de las nuevas generaciones, se debe tener especial interés en la formación de estos dentro del aula. Lamentablemente, no todos los docentes universitarios hacen uso de la tecnología en su práctica pedagógica como parte del proceso de formación de sus estudiantes, es por ello, se pretende argumentar mediante un análisis reflexivo sobre lo que está pasando en la universidad en cuanto al uso de los objetos virtuales de aprendizaje en esta era etiquetada como altamente tecnológica. Considerando necesario realizar una contextualización teórica sobre la problemática y poder presentar propuestas de cambio, con la finalidad de transformar el paradigma de la educación tradicional.
Es importante preguntarse ies solo a finales del siglo XX e inicios del XXI que la tecnología ha formado parte de los procesos educativos? o iestamos frente a un continuo cambio de tecnología educativa? La reflexión nace a partir de lo hoy que se considera como la era de la información; sin embargo, no es del todo cierto que el uso de las TIC sea un privilegio exclusivo de la modernidad.

En su libro Enseñar en la Era Digital de Bates (2019) hace un análisis histórico de cómo la educación ha echado mano de tecnología, afirma que "el debate sobre el rol de la tecnología en la educación se remonta por lo menos a 2500 años atrás", considera innovador cómo el ser humano mantuvo su folklore, historias, transmisión de ciencia y conocimiento, entre otros a través de la comunicación oral y del gran salto que dio hacia la escritura, manteniéndose con ésta la esencia exacta de la palabra sin más modificaciones que la que el escribano dispusiera.

El uso de medios físicos como la pizarra donde el profesor asienta la teoría no es más que un perfeccionamiento de la técnica de cincelar la piedra o dibujar en ella de nuestros antepasados, hasta la actualidad en que las pizarras digitales siguen asombrando con sus bondades a niños y adultos. Y qué decir de la computadora nombre derivado del latín computare cuyo significado es calcular, aunque es inútil discutir sobre sus bondades, ya el hombre primitivo calculaba con piedras, y el Quipu (khipu en su lengua de origen) era efectivamente usado por pueblos indígenas andinos como instrumento de almacenamiento de información.

Este endeble viaje histórico motiva a la reflexión sobre el rol de la tecnología en el desarrollo de la ciencia. En este momento se vuelve preciso hablar sobre la universidad pues es el lugar donde la ciencia se crea, desarrolla, modifica y promueve. 
La universidad ocupa un papel fundamental en el cambio de los paradigmas sociales acerca del conocimiento o del saber en general, bien decía el filósofo y ensayista español José Ortega y Gasset (Palma, 2001) "...en la Universidad se cultiva la ciencia misma, se investiga y se enseña de ello...", en resumen es la universidad donde se formarán los futuros formadores.

Desde el punto de vista que considera a la universidad como formadora $y$ transformadora del conocimiento, se vuelve de vital importancia no solo analizar a los docentes como los que guían los procesos de enseñanza-aprendizaje, sino a su vez internarse en las técnicas pedagógicas que utilizan para conseguir sus objetivos y aquí el corazón del presente análisis ¿es efectiva la vinculación intencional y estructuradamente dirigida entre la educación y la tecnología?, si es así ¿Por qué su uso no está extendido? (Gallo, 1961; Litwin, 2000)

\section{Teorías de la reproducción y resistencia}

Para introducirse en la teoría que sustenta la presente revisión se parte de un breve análisis crítico del autor norteamericano Henry Giroux sobre las teorías de la Reproducción y Resistencia en la nueva sociología de la educación.

Giroux describe a la Reproducción como "producir algo y reproducirlo indefinidamente", esto contextualizado a la educación propone que las escuelas son las encargadas de reproducir el conocimiento necesario para desenvolverse en un entorno social, el manejo conceptual sobre el que se cimienta la teoría establece que los roles sociales están preestablecidos, que hay diferentes tipos de clases y que cada una de éstas tienen un fin específico algo así como dominantes y dominados. De aquí nace la segunda teoría la Resistencia o de romper paradigmas, esquemas, moldes, una institución que genera un pensamiento crítico motivando a sus discentes a desechar lo preestablecido, dejando de reproducir lo enseñado para convertirse en un agente de cambio. (Holguín, 2013).

Surge así la pedagogía crítica, sus postulantes principales Paulo Freire y el mismo Giroux hablan sobre la necesidad de establecer una academia más empática con las nuevas generaciones, motivadoras del pensamiento y autocríticas con sus cimientos, y de la forzosa necesidad de no seguir las corrientes simplemente porque si, sino por el contrario de establecer bases sólidas que generen una ciencia sustentable.

\section{Paradigma de las tecnologías educativas}

En este sentir innovador y en pos de garantizar un adecuado proceso pedagógico el nuevo eje paradigmático de las tecnologías educativas busca fundamentar el uso de las TIC en las aulas universitarias cuya función principal es generar competencias profesionales, por lo tanto éstas están sujeta a la constante modernización de sus procesos de enseñanza-aprendizaje, y en esta ola de avance educativo el uso de espacios virtuales tipo laboratorio para fortalecer y consolidar los conocimientos en los estudiantes va teniendo cada día más impulso. Al respecto se pueden citar diversos estudios que demuestran la eficacia del uso de los laboratorios virtuales.

En España Cerezo y Sastrón (2015) realizaron un estudio sobre los laboratorios virtuales y docencia de la automática en la formación tecnológica de alumnos preuniversitarios; trabajaron con dos grupos: a) uno experimental que usó un laboratorio virtual como complemento a sus clases normales, y b) un grupo de control que 
recibió la materia con la misma metodología usada hasta entonces por el docente. Los resultados de dicha investigación arrogaron que "la educación mediante laboratorios virtuales motiva más a los alumnos para realizar el trabajo propuesto. También, desde el punto de vista de los objetivos educativos, se alcanzó en términos generales, mejores resultados".

Fiad y Galarza (2015) de la Universidad Nacional de Catamarca en Argentina, evaluaron la implementación de un laboratorio virtual como estrategia para el proceso de enseñanza-aprendizaje del concepto de Mol; trabajaron con todos los alumnos ingresantes de la cátedra usando un diseño experimental con preprueba $\mathrm{y}$ postprueba, al igual que el estudio anterior se dividió a su población en dos grupos: a) un grupo control (GC), y b) un grupo experimental (GE). Al finalizar concluyó que:

Los estudiantes del GE obtuvieron una diferencia significativa entre el conocimiento de los conceptos involucrados en el tema cantidades atómico-moleculares adquiridos con la intervención didáctica realizada, respecto al conocimiento conceptual con el que se iniciaron; mientras que los del GC, sujetos a una instrucción tradicional, no muestran una diferencia significativa entre el conocimiento conceptual con el que iniciaron respecto al evaluado después de la instrucción.

Como dato relevante el estudio también señaló que al utilizar el factor Hake para determinar la ganancia de los aprendizajes, los estudiantes del GE obtuvieron "un valor comprendido dentro del intervalo reportado en la literatura como satisfactorio, con una ganancia de aprendizaje alta". (Fiad y Galarza, 2015).

En esta misma línea Cabrera, Sánchez y Rojas (2016), estudiaron el uso de Objetos Virtuales de Aprendizaje (OVAs) como estrategia de enseñanza-aprendizaje inclusivo y complementario a los cursos teóricosprácticos en la Universidad Cooperativa de Colombia Sede Neiva; los autores desarrollaron herramientas de investigación que les permitió determinar los efectos en los procesos de enseñanza-aprendizaje al utilizar una plataforma tecnológica complementaria, para ello trabajaron con estudiantes de cuarto semestre de la carrera de Ingeniería de Sistemas. Sus resultados determinaron que:

Con el uso de OVAs se amplía el panorama del trabajo presencial, se puede profundizar en el análisis del problema simulando situaciones reales en un entorno controlado; el cual genera motivación para que profesores y estudiantes exploren otros aspectos relacionados con el aprendizaje en el aula o fuera de ella y resalta el trabajo autónomo-colaborativo y la comunicación entre profesor-estudiante $\mathrm{y}$ estudiante-estudiante $\mathrm{o}$ entre grupos de trabajo para alcanzar aprendizaje.

El estudio destaca que "con el uso de OVAs se rompe el paradigma del uso del aula de clase o el del aula de laboratorio presencial como escenario tradicional para la apropiación de conocimientos". (Cabrera, Sánchez y Rojas, 2016).

El objetivo principal de utilizar estrategias pedagógicas vinculadas a la tecnología debería ser garantizar un óptimo desarrollo de las competencias académicas del estudiante, tal como lo mencionan Fiad y Galarza el "éxito de los estudiantes está relacionado no sólo con sus capacidades intelectuales sino también, con los procedimientos $o$ estrategias que han desarrollado para alcanzar sus objetivos de aprendizajes" (Fiad y Galarza, 2015). En esta corriente, Casillas, Ramírez y Ortega (2016) señalaron que los estudiantes que manejan diferentes herramientas tecnológicas, tienen mayores oportunidades de exponerse a 
interacciones cotidianas de tipo académicas, "...se benefician de las mejores notas, obtienen el reconocimiento de sus profesores y compañeros y logran capitalizar estas ventajas durante su trayectoria escolar".

Por consiguiente, cuando el espacio de enseñanza-aprendizaje es motivador (entendido no solo como el espacio físico sino todos los elementos materiales e inmateriales del entorno educativo), los contenidos serán de fácil consolidación, de ahí que el docente debe ser capaz de utilizar todas las estrategias que le permitan llegar a ese nivel de satisfacción, la motivación por lo tanto juega un rol importante en el ámbito académico, cabe notar que este no es un proceso unitario exclusivamente enmarcado en el aspecto emocional, sino que abarca componentes muy diversos entre ellos las estrategias pedagógicas que usa el docente, permitiendo de esta manera generar y desarrollar "cursos con más recursos de aprendizaje, por lo tanto, más interesantes y dinámicos" (Núñez y González, 1996 cp. Fiad y Galarza, 2015; Abarca, 2015). Esto motivaría de forma dual al docente y a los estudiantes, convirtiéndose en protagonistas con un rol activo de su propio proceso de enseñanza-aprendizaje.

Bien lo señala Abarca (2015), la motivación en el docente determinará el interés de este por actualizarse en herramientas digitales que permitan una enseñanza acorde a los avances tecnológicos, “...el tipo de TIC y su frecuencia de uso va a depender de los conocimientos que posea el docente y las necesidades de contenidos que se necesite enseñar".

En consecuencia la interrogante que surge, se orienta al tipo de estrategias pedagógicas actuales que puede utilizar el docente universitario para fortalecer los aprendizajes en sus estudiantes y así desarrollar mejores competencias, y esta pregunta nace después de que se entiende que el docente ya no es sólo el proveedor de conocimiento, sino también el ente encargado de consolidarlo, además de fomentar los procesos mediante los cuales se usa adecuadamente la ciencia para alcanzar los objetivos que se proponga.

$Y$ qué decir de los estudiantes universitarios, Rodríguez y Espinoza (2017) se planearon esta interrogante y encontraron que estos presentaban inconvenientes para dominar las diferentes plataformas virtuales, esto debido a que se manifiesta escasa participación por parte de la planta docente; por lo tanto en este punto el docente y las autoridades académicas son responsables de velar y capacitar al discente para que tenga un aprovechamiento efectivo de los medios digitales, el mismo que le permitirá adaptarse a la modalidad de aprendizaje virtual, satisfaciendo así las exigencias actuales de la educación. (Cruz y Miranda, 2017).

Bien lo resalta Hernández (2003) citado por Fiad y Galarza "la enseñanza tradicional de pizarrón está en contraposición con el mundo real donde viven los alumnos, porque ha cambiado la forma de interrelacionarse como resultado de las experiencias con la tecnología fuera de la escuela" (Fiad y Galarza, 2015). Así lo reafirma el estudio realizado por Abarca (2015), quien refiere que hoy por hoy se han sumado nuevas herramientas digitales en la impartición de clases como: presentación de videos educativos a través de proyectores, audios, presentaciones de PowerPoint, aulas virtuales, entre otros, que facilitan el contacto entre el docente y el estudiante. Por lo tanto, el docente y en especial el universitario, está obligado a buscar nuevas estrategias pedagógicas para ponerse a la altura de las exigencias modernas facilitado el proceso de enseñanzaaprendizaje dentro del aula.

En este contexto, el uso de espacios tecnológicos va teniendo cada vez más 
adeptos pues estos juegan un papel esencial en la reestructuración y mejoramiento de los procesos de enseñanza-aprendizaje. Integrar este recurso a la docencia universitaria puede convertirse en una estrategia para motivar a los estudiantes pues, entre algunas de sus ventajas están:

- Promover la variedad metodológica.

- Mejorar la accesibilidad a información actualizada.

- Propiciar el óptimo desenvolvimiento del alumno, mejorando su nivel de comprensión y uso de la información.

- Incentivar el trabajo en equipo, mejorando su trabajo individual.

- Crear nexos con otras comunidades educativas de distintos niveles. (Díaz, 2004, Rosado y Herreros, 2009 cp. Fiad y Galarza, 2015)

No cabe duda después de revisar los estudios que el uso de la tecnología como parte de la docencia universitaria es fundamental en esta era de la información, surge así la siguiente pregunta ¿Por qué si las TIC son efectivas los docentes no las usamos a plenitud?, ¿Qué justifica este deficiente desempeño tecnológico por parte de los docentes?

\section{Enseñar a nativos digitales}

Citando a Rosler (2011) "yo aprendí ayer con información de antes de ayer y tengo que enseñarles a estudiantes que están viviendo en el futuro y eso es muy complejo". Los docentes están siendo avasallados día tras día por una ola insaciable de nativos digitales, estudiantes que nacieron en la era digital y tienen un dominio empírico de las TIC, en palabras de Rosler:

...los docentes somos inmigrantes digitales, con lo que la informática es nuestra segunda lengua y los alumnos se dan cuenta porque se nota en nuestro acento, a diferencia de [ellos] en los que la informática es su lengua materna que la viene hablando desde la cuna. (Rosler, ob. cit., s/p)

La docencia universitaria ya no se suscribe al aula física y mucho menos a la charla magistral, es transcendental la alfabetización tecnológica y aquí viene un punto a favor de los docentes pues aunque los estudiantes manejan muy bien las TIC, aquellas que se usan para apoyar su formación académica todavía les es nueva. Por lo tanto los espacios virtuales que usan los profesores como parte de los procesos pedagógicos deben responder a la diversidad no solo de estilos de enseñanza-aprendizaje sino de recursos tecnológicos disponibles y así aumentar la posibilidad de éxito académico. (Rodríguez, 2011)

Sin embargo, usar la tecnología no significa exclusivamente hacer presentaciones en PowerPoint con infinidad de letras, o poner videos de YouTube, ni mucho menos llenar las aulas virtuales de textos en PDF, pues ¿Cuál sería la diferencia con la clase tradicional? Rodríguez R., en su artículo nos hace reflexionar sobre esto al decir que "la integración de las TIC en modelos formativos no adecuados no solo no mejora el aprendizaje sino que lo empeora incrementando la carga del profesorado y de los estudiantes", el objetivo de las TIC no es volver al estudiante en un actor pasivo consumidor de ideas, sino por el contrario un ente generador y cooperante de conocimiento, pero ¿cooperante con quién?, con su profesor. (Rodríguez, 2011)

Cruz y Miranda (2017) refieren que el $80.66 \%$ de los estudiantes manejan medios virtuales y digitales en el ambiente académico diariamente, convirtiéndose en una fortaleza 
que los docentes deben potenciar ya que es considerada como favorecedora, "lo que conduce a situar estas habilidades en beneficio de su desarrollo académico, además de aprovechar sus bondades para comunicación, investigación, compartir conocimiento y experiencias".

\section{Tecnofilia, Tecnofobia $y$ resistencia digital}

Abordar la Tecnofilia, Tecnofobia y Resistencia Digital por separado asumiendo que solo los docentes sufren de cualquiera de estos "males" es erróneo, es por esto que se hablará de cada uno de ellos respetando las particularidades que presentan los docentes y alumnos.

La Tecnofilia se entiende como la convicción casi inequívoca de que las TIC son la solución pedagógica a todos los problemas de la academia, el docente asume el rol de proveedor exclusivo de tecnología y el discente su consumidor nato, se hace caso omiso del valor del contacto humano y se podría llegar al extremo de trabajar en una burbuja virtual. Su contraparte la Tecnofobia como cualquier otro miedo rechaza el contacto con la fuente productora de su temor, sus defensores consideran deshumanizantes las técnicas pedagógicas que usan a las TIC; los dos grupos por decirlo así, intentan contagiar con sus ideas al resto. (Fuentes, y Ortiz, 2004)

Esta antinomia de concepciones no es fácil de vencer y sin duda alguna quizás este es el origen de la resistencia digital de un tercer grupo, aquellos que no quieren lidiar con las TIC aunque reconocen sus ventajas y desventajas se mantienen "neutrales", la razón principal de esta actitud es el no querer complicarse más de lo debido.

\section{¿Qué hacer para romper las barreras creadas sobre el uso de las TIC?}

Cabe recalcar que las TIC ya forman parte de docencia universitaria, puede citarse lo común que se han vuelto las plataformas Moodle, clínicas de simulación, laboratorios virtuales, entre otras; sin embargo, éstas están sobrevaloradas pues aún no se generan las competencias necesarias para usarlas a cabalidad, si esto no se corrige a tiempo tal como lo mencionan Gutiérrez, Palacios y Torrego (2010) "La integración curricular de las TIC sin la necesaria reflexión desde postulados educativos nos llevaría a convertirlas en refuerzo de un modelo reproductor, unidireccional y vertical de la enseñanza como transmisión de contenidos", para decirlo de otra manera usar lo viejo camuflado con lo nuevo.

Antes de nada hay que empezar a reconocer el valor que las TIC tienen siempre y cuando se articulen con una adecuada estrategia pedagógica, no se puede desestimar el valor que el docente añade a sus clases pues ¿cuál es el valor que la tecnología agrega a la docencia si no hay hilo conductor entre ésta y los estudiantes?, y aquí el reto, los docentes han de garantizar a través de estructuras o procesos pedagógicos bien cimentados que el uso de la tecnología no sea una "moda" más, sino por el contrario una poderosa arma de consolidación de aprendizajes.

"La interactividad es una actividad recíproca, es una comunicación de doble vía, que puede ser física o mental y que se produce entre personas y/o aparatos" (Montero, 1995). La interacción por sí sola no opera, necesita de una retroalimentación que mejore la trasmisión de mensajes entre el trasmisor y el receptor y más aun con correcta aplicación de materiales electrónicos interactivos que permitan un cierto grado de control sobre el proceso de aprendizaje ya 
que deja autonomía en los aprendices en su nivel de interactividad. Así mismo, el material es adaptativo que permite adecuar según el tipo de aprendizaje en lo educandos.

\section{METODOLOGÍA}

Este documento nace después de realizar una indagación cuidadosa mediante metabuscadores y plataformas digitales como Google Scholar, Redalyc, Dialnet, Scielo Ecuador, entre otras acerca del uso de la tecnología de información comunicación (TIC) en las aulas universitarias, para ello se buscó información de investigaciones afines con la temática de los últimos 10 años, además del correspondiente soporte bibliográfico.

\section{DISCUSIÓN}

Es necesario partir de la puesta en práctica de la Pedagogía, misma que según Alirio Liscano se centra en el acto de enseñar, o mejor dicho, cómo enseñar. (Liscano, 2007).

La pedagogía o esta ciencia de la educación ha sufrido a lo largo de la historia muchos cambios, desde la época en la que el profesor era el único poseedor de la ciencia y aquel capaz de entenderla y aplicarla, hasta la actualidad en la que los procesos de enseñanza son más participativos e inclusivos y la relación profesor-estudiante es cada vez más estrecha y horizontal. La práctica pedagógica ya no se limita al traspaso de la información sino más bien a la relación cooperativa del aprendizaje.

$\mathrm{Al}$ respecto, Romero (2009) señala que "la pedagogía es una ciencia de carácter psicosocial que tiene por objeto el estudio de la educación con el fin de conocerla, analizarla y perfeccionarla".

El ejercicio de la pedagogía en las aulas universitarias, ha hecho necesaria la implementación de nuevas estrategias de enseñanza-aprendizaje; el escenario actual de la educación debe estar alineado con las tendencias tecnológicas, es cada vez más necesario echar mano de los avances informáticos.

El objetivo principal de utilizar nuevas estrategias pedagógicas debería ser garantizar un óptimo desarrollo de las competencias académicas del estudiante, tal como lo mencionan Fiad y Galarza el "éxito de los estudiantes está relacionado no sólo con sus capacidades intelectuales sino también, con los procedimientos o estrategias que han desarrollado para alcanzar sus objetivos de aprendizajes". (Fiad y Galarza, 2015)

El análisis realizado en este documento permitió determinar que el uso de la tecnología se ha vuelto un pilar fundamental de la docencia, las universidades de prestigio internacional han marcado claramente la ruta a seguir al usar como elemento cardinal a las TIC. Cada día se fomenta la vinculación que debe tenerse con los objetos virtuales de aprendizaje; no obstante no se puede dejar de lado el aspecto humano, el docente es quien da vida a la academia, ninguna tecnología reemplaza el contacto directo y la retroalimentación in situ que el maestro da, y esto sin tomar en cuenta la riqueza social, cultural, emocional, etc., que el espacio compartido en el aula de clase genera.

\section{A MANERA DE REFLEXIÓN FINAL}

La evolución de la pedagogía obliga al docente a actualizar sus estrategias de enseñanza, ya no se puede seguir desvinculando a la universidad de las TIC.

No cabe duda alguna del beneficio del uso de los objetos virtuales en la docencia universitaria, estamos en una era tecnológica y esto innegablemente nos pone más cerca del conocimiento, que bien utilizado resulta en favor de la sociedad.

Aunque las TIC son evidentemente útiles, es imposible dejar de lado al docente, pues es este el que a través de su "toque especial" puede satisfacer las necesidades de sus 
estudiantes, es por lo tanto indispensable formarlo y proveerle de todos los medios necesarios para que pueda echar mano de la tecnología.

\section{REFERENCIAS}

Abarca Amador, Y. (2015). El uso de las TIC en la educación universitaria: motivación que incide en su uso y frecuencia. Revista de lenguas modernas, (22) (335-349). ISSN: 1659-1933. Costa Rica

Bates, T. Enseñar en la Era Digital: Guía para el diseño de la enseñanza y el aprendizaje. Recuperado el 18 de abril de 2019, de https://cead.pressbooks.com/

Cabrera, J., Sánchez, I., y Rojas, F. (2016). Uso de objetos virtuales de aprendizaje OVAS como estrategia de enseñanza aprendizaje inclusivo y complementario a los cursos teóricos - prácticos. Una experiencia con estudiantes del curso física de ondas. Revista Educación en Ingeniería 11 (22). Recuperado el 10 de marzo de 2019, de https://www.educacioneningenieria.org/ index.php/edi/article/view/602

Casillas, Ramírez y Ortega. (2016). Afinidad tecnológica de los estudiantes universitarios. Innovación educativa (México, DF), 16(70), 151-175. Recuperado en 12 de enero de 2020, de http://www.scielo.org.mx/scielo.php?scr ipt=sci_arttext\&pid=S1665-

26732016000100151\&lng=es\&tlng=es

Cerezo, F., Sastrón, F. (2015). Laboratorios virtuales y docencia de la automática en la formación tecnológica de base de alumnos preuniversitarios. Revista Iberoamericana de Automática e Informática industrial 12 (2015). Recuperado el 06 de marzo de 2019, de https://core.ac.uk/download/pdf/81170 720.pdf

Cruz y Miranda. (2017). ICT in University Students of Tourism at Universidad Autónoma De Baja California, México. El periplo sustentable, (33), 528-563. Recuperado en 12 de enero de 2020, de http://www.scielo.org.mx/scielo.php?scr ipt=sci_arttext\&pid=S1870-

90362017000200528\&lng=es\&tlng=en.
Fiad, S., y Galarza, O. (2015). El laboratorio virtual como estrategia para el proceso de enseñanza-aprendizaje del concepto de Mol. Sistema de Información Científica Redalyc 8(4). Recuperado el 05 de marzo de 2019, de https://www.redalyc.org/articulo.oa?id= 373544191011

Fuentes, J. y Ortiz, M. (2004). Una aproximación a la antinomia tecnofobia versus tecnofilia docente. Editorial Universidad de Granada. Recuperado el 25 de mayo de 2019 de http://revistaseug.ugr.es/index.php/publ icaciones/article/view/2298/2416

Gallo, J. (1961). Esquema y objetivos de la Universidad Contemporánea. Anales de la Facultad de Ciencias Jurídicas y Sociales 3 (3). Recuperado el 25 de abril de 2019 de https://revistas.uchile.cl/index.php/ACJY S/article/view/10264/10320

Gutiérrez, A. Palacios, A. y Torrego, L. (2010). Tribus digitales en las aulas universitarias. Comunicar, XVII (34). Recuperado el 15 de junio de 2019 de https://www.redalyc.org/articulo.oa?id= 158/15812481020

Holguín, P. (2013). Teoría y resistencia en educación, hacia una pedagogía crítica desde los planteamientos de Henry Giroux. En clave social 2. (1). Recuperado el 15 de junio de 2019 de http://repository.lasallista.edu.co:8080/ ojs/index.php/EN-

Clave/article/view/497/280

Liscano, A. (2007). La pedagogía como ciencia de la educación. Archipiélago. Revista cultural de nuestra América 14(56). Recuperado el 05 de marzo de 2019, de http://revistas.unam.mx/index.php/arch ipielago/article/view/19931

Litwin, E. (2000). Tecnología educativa. Política, historias, propuestas. Recuperado el 25 de abril de 2019 de https://cursa.ihmc.us/rid=13049069115 62_1271457301_25975/30LIGUORI-

Laura-Las-nuevas-tecnologias.pdf

Montero, P. (1995). Interactividad versus retroactividad, RED, 12, 10-18.

Núñez, J. C., y González-Pumariega, S. (1996). Procesos motivacionales y aprendizaje. 
Psicología de la instrucción, 2, 33-64.

Palma, R. (2001). Misión de la Universidad. Recuperado el 25 de abril de 2019, de http://www.esi2.us.es/ fabio/mision.pdf

Rodríguez, y Espinoza. (2017). Trabajo colaborativo y estrategias de aprendizaje en entornos virtuales en jóvenes universitarios. RIDE. Revista Iberoamericana para la Investigación y el Desarrollo Educativo, 7(14), 86-109. https://dx.doi.org/10.23913/ride.v7i14. 274

Rodríguez, R. (2011). Repensar la relación entre las TIC y la enseñanza universitaria: Problemas y soluciones. Profesorado.
Revista de Currículum y Formación de Profesorado, 15(1). Recuperado el 20 de mayo de https://www.redalyc.org/articulo.oa?id= 567/56717469002

Romero, G. (2009). La pedagogía en la educación. Revista Digital. Innovación y Experiencias Educativas 1(9). Recuperado el 05 de marzo de 2019, de https://www.academia.edu/6504012/LA _PEDAGOGIA_EN_LA_EDUCACI\%C3\%93N _AUTOR\%C3\%8DA_GUSTAVOADOLFO_ROMERO_BAREA_TEM\%C3\%81 TICA_PEDAGOGIA_ETAPA_EI_EP_ESO_BA CHILLERATO_Resumen 